\title{
Investigation of glucose-modified liposomes using polyethylene glycols with different chain lengths as the linkers for brain targeting
}

This article was published in the following Dove Press journal:

International Journal of Nanomedicine

5 January 2012

Number of times this article has been viewed

\section{Fulan $\mathrm{Xie}^{1, *}$ \\ Nian Yaol,* \\ Yao Qin' \\ Qianyu Zhang' \\ Huali Chen' \\ Mingqing Yuan' \\ Jie Tang' \\ Xiankun $\mathrm{Li}^{\prime}$ \\ Wei Fan' \\ Qiang Zhang ${ }^{2}$ \\ Yong $\mathrm{Wu}^{\prime}$ \\ Li Hai' \\ Qin $\mathrm{He}^{\prime}$}

'Key Laboratory of Drug Targeting and Drug Delivery Systems,

West China School of Pharmacy,

Sichuan University, Chengdu, People's

Republic of China; ${ }^{2}$ State Key

Laboratory of Natural and Biomimetic

Drugs, School of Pharmaceutical

Sciences, Peking University, Beijing,

People's Republic of China

*These authors contributed equally to this work

Correspondence: Qin He

West China School of Pharmacy, Sichuan

University, No. 17, Block 3,

Southern Renmin Road, Chengdu,

Sichuan, 61004I, China

Tel +8628 85502532

Email qinhe@scu.edu.cn

Li Hai

West China School of Pharmacy,

Sichuan University, No. 17, Block 3.

Southern Renmin Road, Chengdu,

Sichuan, 6I004I, China

Tel +862885503666

Email smile.hl@I63.com
Background: An intimidating challenge to transporting drugs into the brain parenchyma is the presence of the blood-brain barrier (BBB). Glucose is an essential nutritional substance for brain function sustenance, which cannot be synthesized by the brain. Its transport primarily depends on the glucose transporters on the brain capillary endothelial cells. In this paper, the brain-targeted properties of glucose-modified liposomes using polyethylene glycols with different chain lengths as the linkers were compared and evaluated to establish an optimized drug-delivery system.

Methods: Coumarin 6-loaded liposomes (GLU200-LIP, GLU400-LIP, GLU1000-LIP, and GLU2000-LIP) composed of phospholipids and glucose-derived cholesterols were prepared by thin-film dispersion-ultrasound method. The BBB model in vitro was developed to evaluate the transendothelial ability of the different liposomes crossing the BBB. The biodistribution of liposomes in the mice brains was identified by in vivo and ex vivo nearinfrared fluorescence imaging and confocal laser scanning microscopy and further analyzed quantitatively by highperformance liquid chromatography.

Results: Glucose-derived cholesterols were synthesized and identified, and coumarin 6-loaded liposomes were prepared successfully. The particle sizes of the four types of glucose-modified liposomes were around or smaller than $100 \mathrm{~nm}$ with a polydispersity index less than 0.300 . GLU400-LIP, GLU1000-LIP, and GLU2000-LIP achieved higher cumulative cleared volumes on BBB model in vitro after 6 hours compared with GLU200-LIP $(P<0.05)$ and were significantly higher than that of the conventional liposome $(P<0.001)$. The qualitative and quantitative biodistribution results in the mice showed that the accumulation of GLU1000-LIP in the brain was the highest among all the groups $(P<0.01$ versus LIP).

Conclusion: The data indicated that GLU400-LIP, GLU1000-LIP, and GLU2000-LIP all possess the potential of brain targeting, among which GLU1000-LIP, as a promising drug-delivery system, exhibited the strongest brain delivery capacity.

Keywords: glucose, polyethylene glycols, liposome, BBB, brain-targeted

\section{Introduction}

Some of the most dangerous threats to health are seen in the dramatic increase in incidence and low recovery rate for central nervous system (CNS) diseases such as brain tumor, epilepsy, Alzheimer disease, Parkinson's disease, and neurodegenerative disorders. The presence of the blood-brain barrier (BBB) is a prohibitive obstacle to transporting drugs into the brain parenchyma. Only $2 \%$ of small molecules can pass the BBB through paracellular or transcellular pathways, while the take-up of most of the small molecules and all of the large molecules is prevented by the BBB. ${ }^{1,2}$ Many drugs are unable to reach the action site with an adequate amount in clinical practice because 
of the BBB. Accordingly, the development of brain-targeted drug-delivery systems is imperative.

The unique functional structure of the BBB is composed of brain capillary endothelial cells (BCECs), astrocytes (ACs), and the basement membrane between BCECs and ACs. The BBB helps to maintain homeostasis in the brain, and tight junctions exist between BCECs. ${ }^{3}$ The structural property of the BBB guarantees against the uptake of both harmful substances and therapeutic drugs into the brain parenchyma. Briefly, the BBB not only plays an important role in protecting the brain, but also inhibits drugs from reaching the action site in the brain. ${ }^{4}$

One pathway for substances to cross the BBB is by carrier-mediated transport. The tight junctions effectively switch on BCECs, and the endothelial cells are required to maintain a high level of expression of transporters for essential polar metabolites to facilitate their entry into the brain, such as glucose and amino acids. ${ }^{5}$ The expressions of these carriers were often polarized to optimize substrates (endogenous substances and nutrients) transporting into the brain. Several carrier systems have been described previously, including those specifically designed with small-molecule peptides, hexoses, monocarboxylic acids, amino acids, organic anions, and cations. ${ }^{6-9}$ Full utilization of these carrier systems expressed on the BBB may be an attractive strategy for therapeutic delivery of drugs that otherwise have minimal access to the CNS.

One example of carrier-mediated transporter is hexose transporters, especially glucose transporters (GLUTs). The brain cannot synthesize or store glucose by itself; therefore the glucose transporters are responsible for transporting glucose into or out of the brain internal environment to sustain the cerebral function. A family of GLUT isoforms named GLUT1-7 have been gradually discovered. ${ }^{10,11}$ GLUT1 and GLUT3 are predominant GLUTs on the human BBB. GLUT1 is highly expressed on the membrane of BCECs and responsible for stereoselectively transporting the D-glucose enantiomer into the extracellular space of the brain. ${ }^{12,13}$ GLUT3 is the major neuronal GLUT and helps transport glucose from the extracellular space into the neurons. ${ }^{14}$ In addition, GLUT2 is expressed on the ACs of the brain. Since GLUTs can facilitate the glucose transport from the blood to the brain, glucose analogs should be good candidates for drug transport through the BBB. ${ }^{15,16}$ In previous studies, different glycol-conjugates as candidates for the treatment of CNS diseases have been synthesized, which could increase BBB permeability through the affinity with GLUT1, such as dopamine, ${ }^{17,18}$ glycosyl-derivatives of ibuprofen, ${ }^{19}$ among others. Besides the prodrugs, glucose-modified liposomes using ethylene glycols with short chain length as the linkers have also been investigated. ${ }^{20}$

Polyethylene glycol (PEG)-modified liposomes with long-circulating characteristics are successfully applicable for many delayed and controlled formulations research. The liposomes modified by PEGs can prevent themselves from getting trapped by the reticuloendothelial system (RES) such as liver and spleen. ${ }^{21}$

PEGs with longer chain length may decrease the brain-targeted efficiency of the liposome because of the steric hindrance, while PEGs with shorter chain length may obstruct the exposure of the glucose. In order to explore their difference and determine the optimal length of PEG for drug delivery in this study, the characteristics of glucose-modified liposomes using PEGs with different chain lengths as the linkers were compared and evaluated both in vitro and in vivo, thus developing the optimal brain-targeting drug-delivery system with a strong transendothelial ability crossing the BBB. Glucose residue-linked cholesterol via PEGs with different chain lengths at the C-6 position of the glucose were synthesized, which may theoretically attain high affinity with GLUT1. The glucose-modified liposomes were prepared with these glucose-linked cholesterol derivates. In addition, the brain-delivery properties of these new drug-delivery systems were illustrated in vitro and in vivo. Finally, the brain distribution characteristics of these glucose-modified liposomes were also investigated.

\section{Materials and methods}

\section{Reagents and chemicals}

Soybean phospholipids and cholesterol (CHO) were purchased from Bio Life Science and Technology Co, Ltd (Shanghai, People's Republic of China). Coumarin 6 was purchased from Sigma-Aldrich (St Louis, MO). 1,1'dioctadecyl-3,3,3',3'-tetramethylindotricarbocyanine (DIR) was purchased from Biotium (Hayward, CA). Other chemicals were obtained from commercial sources of analytical grade.

\section{Animals}

Kunming mice were purchased from the Experimental Animal Center of Sichuan University (Sichuan, People's Republic of China). All animal procedures for this study were approved by the Experiment Animal Administrative Committee of Sichuan University. 


\section{Synthesis of CHO-PEG200-GLU, CHO-PEG400-GLU, CHO-PEG I000-GLU, and CHO-PEG2000-GLU}

The potential ligands $\mathbf{L a}-\mathbf{L d}$ were synthesized as outlined in Scheme 1. These ligands possess bifunctional properties: a lipophilic steriod for the insertion into the liposomal membrane and a hydrophilic glucose residue outside the membrane for the recognition of GLUT1. The cholesterol links glucose residue via PEGs with different chain lengths (PEG200, PEG400, PEG1000, and PEG2000) at the C-6 position of the glucose. PEGs with adequate space length could leave the glycosyl group at the liposome surface and provide good anchors for biological receptors and ligands, reducing the nonspecific binding of proteins and other bioactive molecules.

\section{Synthesis of the compound 2}

To the solution of cholesterol $(100 \mathrm{~g}, 260 \mathrm{mmol})$ in $300 \mathrm{~mL}$ dry pyridine, paratoluensulfonyl chloride $(74 \mathrm{~g}, 400 \mathrm{mmol})$ was added at $0^{\circ} \mathrm{C}$. The reaction mixture was stirred at $50^{\circ} \mathrm{C}$ overnight, and then concentrated in vacuum to form residue. The residue was partitioned with ether and water (1:1 $\mathrm{v} / \mathrm{v})$. The aqueous layer was then extracted with ether. The combined organic layer was dried on anhydrous magnesium sulfate and concentrated. The crude product was recrystallized in petrol ether to afford a white solid (116 g, 83\%), and the melting point was $132^{\circ} \mathrm{C}-133^{\circ} \mathrm{C}$.

\section{Synthesis of the compound 3}

The prepared cholesteryl tosylate $2(100 \mathrm{~g}, 185 \mathrm{mmol})$ was refluxed for 5 hours with tetraoxatetradecane diol (176 g, $740 \mathrm{mmol}$ ) in $350 \mathrm{~mL}$ dry dioxane. The insolubles were removed by filtration. The filtrate was concentrated in vacuum, and the residue was redissolved in dichloromethane, washed with brine, dried over anhydrous magnesium sulfate, and concentrated on a rotary evaporator. The residue was purified on a silica-gel chromatography column to get product 3 (44.8 g, 43\%).

\section{Synthesis of the compound 4}

To a solution of alcohol $3(30 \mathrm{~g}, 53.4 \mathrm{mmol})$ and triethylamine (27 g, $267 \mathrm{mmol}$ ) in tetrahydrofuran (THF) in ice bath, methanesulfonyl chloride $(7.9,69.4 \mathrm{mmol})$ was added. After 10 minutes, the solution was warmed to room temperature and further stirred for 4 hours, and the reaction mixture was concentrated. The residue was partitioned with ethyl acetate and water. The aqueous layer was then extracted with ethyl acetate. The combined organic layer was dried on anhydrous magnesium sulfate and concentrated. The crude product was purified on a silica-gel chromatography column to get $\mathbf{4}$ as yellow oil (31.4 g, 92\%).

General procedure for the preparation of compound 5 A solution of 4 (1 equiv) in THF was added dropwise to a slurry of $\mathrm{NaH}$ ( 2 equiv) in THF, and stirred for

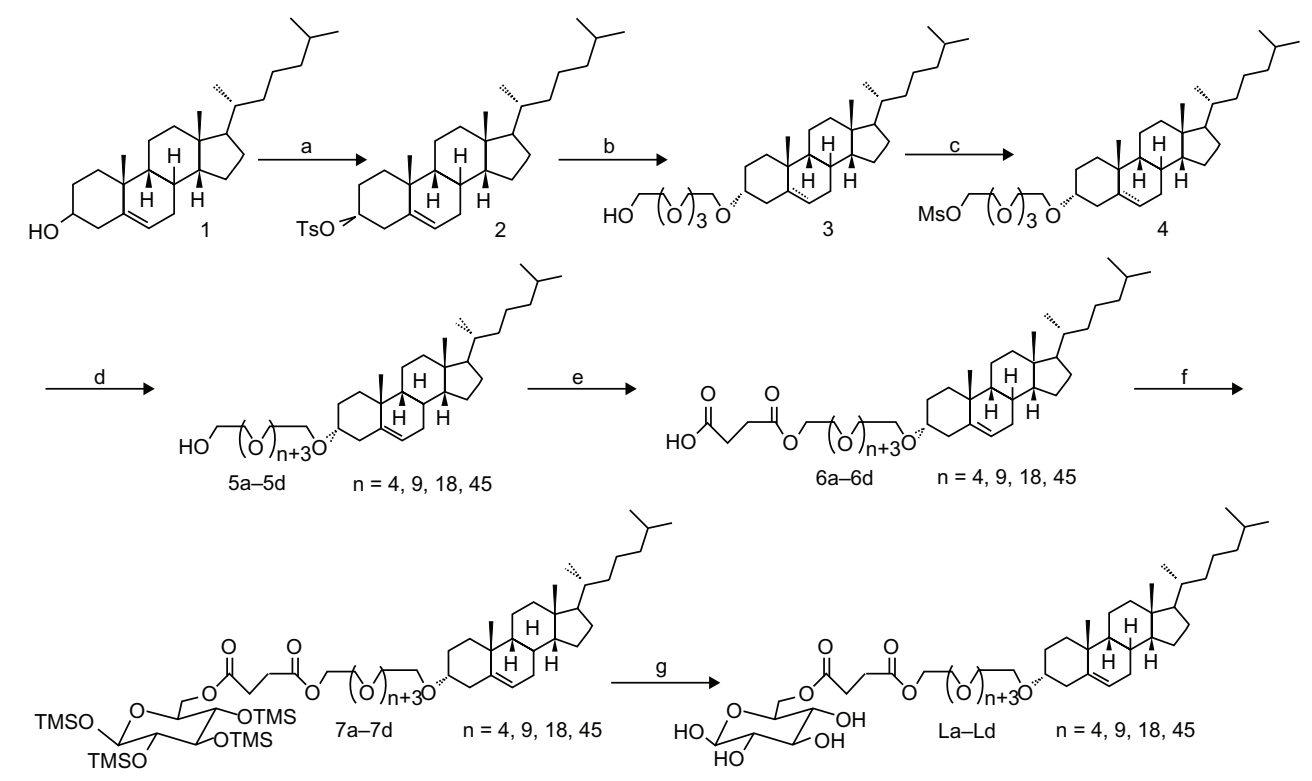

Scheme I Reagents and conditions: (a) TsCl, pyridine, $50^{\circ} \mathrm{C}$, overnight; (b) $\mathrm{HOCH}_{2}\left(\mathrm{CH}_{2} \mathrm{OCH}_{2}\right)_{3} \mathrm{CH}_{2} \mathrm{OH}$, dioxane, reflux, 8 hours; (c) MsCl, Et ${ }_{3} \mathrm{~N}$, THF, rt, 4 hours; (d) PEGs (PEG200, PEG400, PEG I000, PEG2000), NaH, THF, reflux, overnight; (e) succinic anhydride, pyridine, $75^{\circ} \mathrm{C}, 8$ hours; (f) DCC, DMAP, I,2,3,4-tetra-O-trimethylsilylD-glucopyranose, dichloromethane, rt, overnight; (g) TFA, THF, rt, I hour.

Abbreviations: TsCl, 4-toluenesulfonyl chloride; THF, tetrahydrofuran; DCC, dicyclohexylcarbodiimide; DMAP, 4-dimethylaminopyridine; TFA, trifluoroacetic acid. 
another 30 minutes. The PEGs (2 equiv) in THF was dropped in the mixture and refluxed overnight, and then the water was carefully quenched. The mixture was diluted with ethyl acetate and repeatedly washed with brine, dried over anhydrous magnesium sulfate, filtered, and evaporated to dryness. The crude oil was chromatographically purified on a silica column to afford compound $\mathbf{5}$ as yellow oil: 5a (2.43 g, 43\%), 5b (2.87 g, 39\%), 5c (4.07 g, 41\%), and 5d (7.39 g, 38\%).

\section{General procedure for the preparation of compound 6}

To a solution of 5 ( 1 equiv) in pyridine was added succinic anhydride (1.5 equiv). This mixture was stirred at $75^{\circ} \mathrm{C}$ for 8 hours, and then was concentrated. The residue was partitioned with ethyl acetate and water. The organic layer was washed with water, dried on anhydrous magnesium sulfate, and concentrated. The crude product was purified on a silica-gel chromatography column to get acid $\mathbf{6}$ as yellow oil: 6a (1.21 g, 74\%), 6b (1.63 g, 81\%), 6c (3.01 g, 76\%), and $6 \mathbf{d}(4.19 \mathrm{~g}, 64 \%)$.

\section{General procedure for the preparation of compound 7}

A stirred solution of acid 6 (1 equiv) in dry $\mathrm{CH}_{2} \mathrm{Cl}_{2}$ was treated with 1,2,3,4 -tetra- $O$-(trimethylsilyl)-Dglucopyranose (1.1 equiv), ${ }^{21}$ dicyclohexylcarbodiimide (DCC) (1.2 equiv) and 4-dimethylaminopyridine (DMAP) ( 0.1 equiv). The mixture was kept at room temperature overnight and then filtered. The filtrate was concentrated and the residue was purified by flash chromatography to yield 7 as oil: 7a (528 mg, 61\%), $7 \mathbf{b}$ (672 mg, 66\%), 7c (711 mg, 52\%), 7d (803 mg, 42\%).

\section{General procedure for the preparation of compound $\mathrm{L}$}

The compound 7 (1 equiv) in $30 \mathrm{~mL}$ tetrahydrofuran was treated with trifluoracetic acid ( 0.1 equiv) for 1 hour at room temperature and then solid sodium carbonate ( 0.1 equiv) was added. The reaction mixture was filtered and concentrated. The residue was purified by flash chromatography to give $\mathbf{L}$ as oil.

La (492 mg, 82\%), IR (KBr): 3471, 2972, 2938, 1753, 1252, $1114 \mathrm{~cm}^{-1},{ }^{1} \mathrm{H}$ NMR (400 $\left.\mathrm{MHz}, \mathrm{CDCl}_{3}, \delta \mathrm{ppm}\right)$ : 5.33 (m, 1H, chol H-6), 5.27 (m, 1H, H-1), 4.82 (m, 1H, H-6), 4.39 (m, 1H, H-2), 4.26 (m, 1H, H-5), 3.99 (m, 1H, $\mathrm{H}-4), 3.72-3.68$ (m, PEG200-H and $\left.4 \times \mathrm{CH}_{2} \mathrm{CH}_{2} \mathrm{O}\right), 3.54$ (m, 1H, H-3), 3.27 (m, 1H, H-6'), 3.18 (m, 1H, chol H-3),
2.67 (m, 4H, $\mathrm{COCH}_{2} \mathrm{CH}_{2} \mathrm{CO}$ ), 2.37-0.66 (remaining chol protons) with $0.66\left(\mathrm{~s}, 3 \mathrm{H}, \mathrm{CH}_{3}-18\right), 0.85$ (d, 6H, $\mathrm{CH}_{3}-26$, $\left.\mathrm{CH}_{3}-27, \mathrm{~J}=6.7 \mathrm{~Hz}\right), 0.91\left(\mathrm{~d}, 3 \mathrm{H}, \mathrm{CH}_{3}-21, \mathrm{~J}=6.8 \mathrm{~Hz}\right), 0.99$ (s, 3H, $\left.\mathrm{CH}_{3}-19\right)$

Lb (631 mg, 73\%), IR (KBr): 3472, 2972, 2937, 1751, 1251, $1111 \mathrm{~cm}^{-1},{ }^{1} \mathrm{H}$ NMR (400 MHz, $\left.\mathrm{CDCl}_{3}, \delta \mathrm{ppm}\right)$ : 5.33 (m, 1H, chol H-6), 5.25 (m, 1H, H-1), 4.80 (m, 1H, H-6), 4.40 (m, 1H, H-2), 4.25 (m, 1H, H-5), 4.01 (m, 1H, $\mathrm{H}-4), 3.72-3.63$ (m, PEG400-H and $\left.4 \times \mathrm{CH}_{2} \mathrm{CH}_{2} \mathrm{O}\right), 3.52$ (m, 1H, H-3), 3.35 (m, 1H, H-6'), 3.18 (m, 1H, chol H-3), 2.68 (m, 4H, $\mathrm{COCH}_{2} \mathrm{CH}_{2} \mathrm{CO}$ ), 2.37-0.67 (remaining chol protons) with 0.67 (s, 3H, $\left.\mathrm{CH}_{3}-18\right), 0.86$ (d, 6H, $\mathrm{CH}_{3}-26$, $\mathrm{CH}_{3}-27, \mathrm{~J}=6.7 \mathrm{~Hz}$ ), 0.91 (d, $\left.3 \mathrm{H}, \mathrm{CH}_{3}-21, \mathrm{~J}=6.8 \mathrm{~Hz}\right), 0.99$ (s, 3H, $\left.\mathrm{CH}_{3}-19\right)$

Lc (825 mg, 76\%), IR (KBr): 3473, 2975, 2935, 1750, 1253, $1109 \mathrm{~cm}^{-1},{ }^{1} \mathrm{H}$ NMR (400 MHz, $\left.\mathrm{CDCl}_{3}, \delta \mathrm{ppm}\right)$ : 5.32 (m, 1H, chol H-6), 5.24 (m, 1H, H-1), 4.58 (m, 1H, H-6), 4.39 (m, 1H, H-2), 4.32 (m, 1H, H-5), 3.99 (m, 1H, $\mathrm{H}-4), 3.73-3.55$ (m, PEG800-H and $\left.4 \times \mathrm{CH}_{2} \mathrm{CH}_{2} \mathrm{O}\right), 3.48$ (m, 1H, H-3), 3.34 (m, 1H, H-6'), 3.15 (m, 1H, chol H-3), 2.69 (m, 4H, $\mathrm{COCH}_{2} \mathrm{CH}_{2} \mathrm{CO}$ ), 2.37-0.66 (remaining chol protons) with $0.66\left(\mathrm{~s}, 3 \mathrm{H}, \mathrm{CH}_{3-} 18\right), 0.86\left(\mathrm{~d}, 6 \mathrm{H}, \mathrm{CH}_{3}-26\right.$, $\left.\mathrm{CH}_{3}-27, \mathrm{~J}=6.7 \mathrm{~Hz}\right), 0.91$ (d, 3H, $\left.\mathrm{CH}_{3}-21, \mathrm{~J}=6.7 \mathrm{~Hz}\right), 0.99$ (s, 3H, $\left.\mathrm{CH}_{3}-19\right)$

Ld (108 mg, 51\%), IR (KBr): 3471, 2971, 2934, 1750, 1249, $1112 \mathrm{~cm}^{-1},{ }^{1} \mathrm{H}$ NMR (400 MHz, $\left.\mathrm{CDCl}_{3}, \delta \mathrm{ppm}\right)$ : 5.24 (m, 1H, chol H-6), 5.20 (m, 1H, H-1), 4.91 (m, 1H, H-6), 4.50 (m, 1H, H-2), 4.21 (m, 1H, H-5), 4.15 (m, 1H, $\mathrm{H}-4), 3.74-3.36$ (m, PEG2000- $\mathrm{H}$ and $\left.4 \times \mathrm{CH}_{2} \mathrm{CH}_{2} \mathrm{O}\right), 3.33$ (m, 1H, H-3), 3.28 (m, 1H, H-6'), 3.08 (m, 1H, chol H-3), 2.57 (m, 4H, $\mathrm{COCH}_{2} \mathrm{CH}_{2} \mathrm{CO}$ ), 2.28-0.58 (remaining chol protons) with $0.58\left(\mathrm{~s}, 3 \mathrm{H}, \mathrm{CH}_{3}-18\right), 0.76\left(\mathrm{~d}, 6 \mathrm{H}, \mathrm{CH}_{3}-26\right.$, $\mathrm{CH}_{3}-27, \mathrm{~J}=6.7 \mathrm{~Hz}$ ), 0.81 (d, $\left.3 \mathrm{H}, \mathrm{CH}_{3}-21, \mathrm{~J}=6.8 \mathrm{~Hz}\right), 0.93$ (s, 3H, $\left.\mathrm{CH}_{3}-19\right)$

\section{Preparation of liposomes}

Glucose-modified liposomes (GLU200-LIP, GLU400-LIP, GLU1000-LIP, and GLU2000-LIP) loaded with coumarin 6 were prepared by the lipid film hydration-ultrasound method with cholesterols modified with PEG linkers of different lengths. Briefly, lipid materials (the molar ratio of phospholipid and cholesterol derivatives was 2:1) and coumarin 6 were dissolved in chloroform, and then chloroform was removed by vacuum evaporation on a rotary evaporator. The uniform thin film was kept under vacuum to remove the organic solvent completely. The lipid film was then incubated in tris-EDTA buffered saline $(10 \mathrm{mM}$ tris, $2 \mathrm{mM}$ EDTA; pH 7.4) for 1 hour at $37^{\circ} \mathrm{C}$ with constant mixing. 
Subsequently it was further intermittently sonicated by a probe sonicator at $80 \mathrm{~W}$ for 50 seconds. The final lipid concentration was $7.1 \mu \mathrm{mol} / \mathrm{mL}$, while the final concentration of coumarin 6 was $4.8 \mu \mathrm{g} / \mathrm{mL}$.

Conventional liposomes were evaluated as the control in this study. Liposome suspensions were diluted with tris-EDTA buffered saline, and the average diameters, polydispersity indices (PDI) and zeta-potentials of LIP, GLU200-LIP, GLU400-LIP, GLU1000-LIP, and GLU2000LIP were determined by Malvern Zetasizer Nano ZS90 (Malvern Instruments Ltd, Malvern, UK) utilizing dynamic light scattering.

Nearinfrared (NIR) fluorescence probe DIR-loaded liposomes employed in the in vivo and ex vivo optical imaging studies were prepared by the method described above, with the probe replaced by DIR. The final concentration of DIR was $20 \mu \mathrm{g} / \mathrm{mL}$.

\section{Stability of coumarin 6-loaded liposomes in the presence of fetal bovine serum} LIP, GLU200-LIP, GLU400-LIP, GLU1000-LIP, and GLU2000-LIP were prepared as described above. One hundred microliters of the liposomes were separately mixed with $1 \mathrm{~mL}$ phosphate-buffered saline containing $10 \%(\mathrm{v} / \mathrm{v})$ fetal bovine serum (FBS) respectively, and the mixtures were kept at $37^{\circ} \mathrm{C}$ with constant slight mixing for 24 hours. The transmittance of each sample was detected at different time points at the wavelength of $750 \mathrm{~nm}$. The variation curves were delineated to investigate the stability of LIP, GLU200LIP, GLU400-LIP, GLU1000-LIP, and GLU2000-LIP.

\section{Transendothelial ability of coumarin 6-loaded liposomes on an in vitro BBB model}

\section{Establishment of the BBB model in vitro}

In the study, the BBB model in vitro was established by coculturing BCECs and ACs. The coculture model has been widely employed in many brain-targeted studies and the culture process of BCECs and ACs has been described previously. ${ }^{22,23}$ After a series of purification, the morphologies of the ACs and BCECs were characterized by inverted fluorescence microscopy. BCECs and ACs were cocultured in a "contact through feet" model. ${ }^{24,25}$ Microporous Transwell membranes with a pore diameter of $1 \mu \mathrm{m}$ were inserted in a 24-well plate and turned upside down, and the ACs at the second passage were cultured on the bottom side of the microporous Transwell membranes at a density of $5 \times 10^{5}$ cells $/ \mathrm{mL}$.
After allowing the ACs to attach for 4 hours, the plate was turned over. The complete culture medium for ACs containing 20\% FBS was added into the wells. Approximately 5 days later, BCECs at the third or fourth passage were seeded onto the intramembrane of the inserts at a density of $2.5 \times 10^{5}$ cells $/ \mathrm{mL}$. The complete culture medium for BCECs containing 20\% FBS was added to the upper side of the membranes. After 5-7 days of coculturing, the transendothelial electric resistance (TEER) value of the cells was measured by a Millicell ERS (Millipore, Bedford, MA).

\section{The fluorescein sodium leakage test} of the BBB model in vitro

The primarily cultured AC was inoculated on the bottom side of microporous Transwell membranes which were inserted in a 24-well plate. After reaching confluence under $37^{\circ} \mathrm{C}$ in $5 \% \mathrm{CO}_{2}$, Ringer-HEPES buffer ( $150 \mathrm{mM} \mathrm{NaCl}, 5.2 \mathrm{mM} \mathrm{KCl}$, $2.2 \mathrm{mM} \mathrm{CaCl}_{2}, 0.2 \mathrm{mM} \mathrm{MgCl}, 6 \mathrm{mM} \mathrm{NaHCO}_{3}, 2.8 \mathrm{mM}$ glucose, and $5 \mathrm{mM}$ HEPES; $\mathrm{pH} 7.4$ ) was added to the acceptor chambers of the 24 -well plate $(1 \mathrm{~mL} /$ well $)$ to replace the AC culture medium, and then $200 \mu \mathrm{L}$ of $2 \mu \mathrm{g} / \mathrm{mL}$ fluorescein sodium solution was added to the donor chambers. The solution in the acceptor chambers was removed to determine the fluorescence values at predetermined time points, and fresh Ringer-HEPES buffer was supplemented for the next measurement.

The same treatment was applied on the BCECs monolayer cells that were inoculated on the top side of microporous Transwell membranes for the BBB model in vitro. The blank inserts were employed as controls. Triplicate samples were performed. Results were expressed as leakage ratio, which further verified the roles of BCECs or ACs in the BBB construction and the reliability of the $\mathrm{BBB}$ model for the transendothelial experiment.

\section{Transendothelial ability of coumarin 6-loaded liposomes on an in vitro BBB model}

The transport study was performed as described previously. ${ }^{19}$ After the establishment of the BBB model in vitro, RingerHEPES buffer was added to the acceptor chambers of the 24-well plate ( $1 \mathrm{~mL} /$ well) and incubated for 15 minutes under $37^{\circ} \mathrm{C}$ in $5 \% \mathrm{CO}_{2}$. And then $200 \mu \mathrm{L}$ of coumarin 6-loaded liposomes from each group in Ringer-HEPES solution with a total lipid concentration of $160 \mu \mathrm{M}$ were added to the donor chambers, respectively. The fluorescence values of coumarin 6 in the acceptor chambers were measured after incubation for 1 hour, 2 hours, 3 hours, 4 hours, 5 hours, and 6 hours by a RF-5301 fluorospectrophotometer $(\mathrm{Ex}=465 \mathrm{~nm}$, 
$\mathrm{Em}=502 \mathrm{~nm}$; Shimadzu, Kyoto, Japan). Triplicate samples were performed, and the results were expressed as cumulative cleared volume to indicate the transendothelial ability of coumarin 6-loaded liposomes. ${ }^{22}$

\section{Biodistribution of the liposomes in vivo Nearinfrared fluorescence probe DIR imaging for in vivo and ex vivo}

The in vivo studies were approved by Sichuan University Animal Ethical Experimentation Committee according to the requirement of the National Act on the Use of Experimental Animals (People's Republic of China).

Whole mice in vivo NIR fluorescence imaging was performed 2, 6, and 12 hours, respectively after intravenous injection of DIR-loaded liposomes at a dose of $0.2 \mathrm{mg} \mathrm{DIR} / \mathrm{kg}$. Mice were anesthetized and placed into an imaging system (Imaging Station IS2000MM; Kodak, Rochester, NY) equipped with a band-pass filter at $770 \mathrm{~nm}$ and a long-pass filter at $830 \mathrm{~nm}$. Images were captured by the charge-coupled device camera embedded in the imaging. Similarly, in the ex vivo optical imaging study, DIR-loaded liposomes were injected intravenously into the mice at a dose of $0.2 \mathrm{mg} \mathrm{DIR} / \mathrm{kg}$, and after 1 hour the mice were perfused from the heart with normal saline and sacrificed. Their whole brains were harvested and imaged as described above.

\section{Confocal laser scanning microscopy}

Coumarin 6-loaded liposomes were adopted for the confocal laser scanning microscopy (CLSM) study. Mice were anesthetized 1 hour after the intravenous injection of the liposomes at a dose of $0.1 \mathrm{mg}$ coumarin $6 / \mathrm{kg}$, then were perfused with normal saline from the heart and sacrificed. The brains were removed and washed in normal saline. The samples were kept in a liquid nitrogen container overnight. On the next day, sections were cut from coronal plane onto a glass slide by a freezing microtome. Two micrograms per milliliter of DAPI was dropped onto the glass slides, and the glass slides were placed for 5 minutes in darkness. After being washed with distilled water, the frozen sections were fixed by glycerol. The fluorescent images were captured by a Leica TCS SP5 AOBS confocal microscopy system (Leica, Solms, Germany).

\section{Quantitative biodistribution study}

Fifteen mice were randomly assigned to five groups of three mice each, and treated by LIP, GLU200-LIP, GLU400-LIP, GLU1000-LIP, and GLU2000-LIP via the tail veins respectively at a dose of $0.1 \mathrm{mg} / \mathrm{kg}$ of coumarin 6 .
Mice were anesthetized 1 hour after the liposome injection, perfused with normal saline in the heart, and sacrificed. Subsequently, organs including hearts, livers, spleens, lungs, kidneys, and brains were harvested and flushed with normal saline to remove the remaining blood. ${ }^{26}$ All the organs were immersed with a triple amount of water and subjected to homogenization. Ten microliters of internal standard (coumarin 7) was precisely added into $200 \mu \mathrm{L}$ organ homogenate, and the mixture was extracted with $1 \mathrm{ml}$ N-hexane. After centrifugation at 10,000 rpm for 5 minutes, the supernatant was collected and dried under an air stream at room temperature. Fifty microliters of methanol was added into the dry residue followed by vortexing and sonicating, and the sample was centrifuged at 12,000 rpm for 10 minutes. Twenty microliters of the supernatant was injected into a high-performance liquid chromatography (HPLC) system for analysis. A reversed-phase HPLC (Alltech, Deerfield, IL) system with a Diamonsil $C_{18}$ column $(200 \times 4.6 \mathrm{~mm}, 5 \mu \mathrm{m})$ was employed for the analysis in which methanol-water (92:8) was used as the mobile phase. The flow rate was $1 \mathrm{~mL} / \mathrm{min}$ and the column temperature was $35^{\circ} \mathrm{C}$. The excitation and emission wavelength of coumarin 6 were $465 \mathrm{~nm}$ and $502 \mathrm{~nm}$, respectively.

\section{Statistical analysis}

SPSS software (v. 11.0; SPSS Inc, Chicago, IL) was applied for the statistical analysis. The comparisons between different groups were investigated by analysis of variance (ANOVA) and unpaired bilateral $t$-test was performed for the assessment of the samples within a group.

\section{Results}

\section{Characterization of glucose-modified liposomes linked by PEGs of different chain lengths}

The electron microscope results showed that the liposomes (GLU200, GLU400, GLU1000, and GLU2000) all exhibited uniform spherical appearance, demonstrating the successful formation of the liposomes (data not shown). As shown in Table 1, the average diameter of the conventional coumarin 6-loaded liposome was around $158 \mathrm{~nm}$ with a PDI of 0.172 , while the average particle sizes of the four types of glucosemodified liposomes were all around or smaller than $100 \mathrm{~nm}$ with PDIs less than 0.300 .

The aggregation characteristics in FBS were investigated subsequently. As shown in Figure 1, the transmittance of LIP was below $90 \%$ after incubation with phosphatebuffered saline containing $10 \%(\mathrm{v} / \mathrm{v})$ FBS for 2 hours. 
Table I The size and Zeta-potential of different coumarin 6-loaded liposomes $(n=3)$

\begin{tabular}{|c|c|c|c|c|}
\hline & Molar ratio of lipids & $\begin{array}{l}\text { Average size } \\
(\mathrm{nm})\end{array}$ & $\begin{array}{l}\text { Polydispersity index } \\
\text { (PDI) }\end{array}$ & $\begin{array}{l}\text { Zeta-potential } \\
(\mathrm{mV})\end{array}$ \\
\hline LIP & PC:cholesterol (2:1) & $158.0 \pm 7.1$ & $0.172 \pm 0.028$ & $-60.4 \pm 12.3$ \\
\hline GLU200-LIP & PC:CHO-PEG200-GLU (2:I) & $86.3 \pm 5.7$ & $0.270 \pm 0.015$ & $-33.3 \pm 3.5$ \\
\hline GLU400-LIP & PC:CHO-PEG400-GLU (2:I) & $81.5 \pm 6.3$ & $0.252 \pm 0.016$ & $-36.2 \pm 2.6$ \\
\hline GLUI000-LIP & PC:CHO-PEGI000-GLU (2:I) & $100.0 \pm 5.0$ & $0.265 \pm 0.032$ & $-24.9 \pm 2.8$ \\
\hline GLU2000-LIP & PC:CHO-PEG2000-GLU (2:I) & $104.9 \pm 8.7$ & $0.289 \pm 0.013$ & $-20.9 \pm 2.4$ \\
\hline
\end{tabular}

Abbreviations: LIP, liposomes; GLU, glucose; PC, phospholipid; $\mathrm{CHO}$, cholesterol.

Compared with LIP, the transmittances of other four types of glucose-modified liposomes were all above $90 \%$ over 24 hours, displaying an improved stability compared with the conventional liposomes.

\section{The transendothelial ability of coumarin 6-loaded liposomes on an in vitro BBB model}

The BBB model in vitro was established by coculturing BCECs and ACs as described above to study the transendothelial ability of the liposomes. In order to better simulate the properties of BBB, we cultured BCECs on the top side of the membrane and the ACs on the bottom side. In the coculturing process, the ecphyma could extend from the $\mathrm{ACs}$ through the membrane pores to contact BCECs.

To authenticate the integrity of the model in vitro, a fluorescein sodium leakage test was performed and TEER was measured during the whole transport process. Fluorescein sodium is a fluorescent compound usually applied in the detection of the tight junctions of cell layers. As shown in Figure 2, the fluorescein sodium leakage test illustrated the integrity of the BBB model and the formation of the tight junctions. Over 6 hours in the coumarin 6-loaded

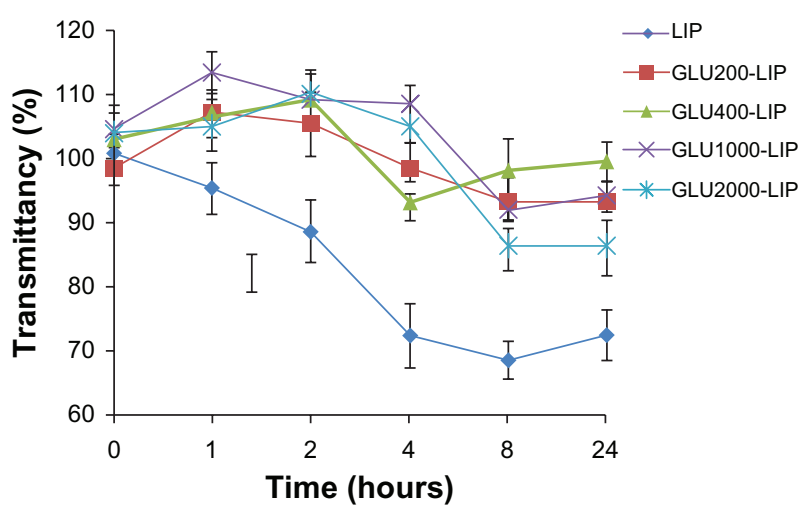

Figure I The variation in transmittancy versus the different incubation time of liposomes at the wavelength of $750 \mathrm{~nm}$ when incubated with phosphate-buffered saline containing $10 \%(v / v)$ fetal bovine serum for 24 hours at $37^{\circ} \mathrm{C}(n=3)$. Abbreviations: GLU, glucose; LIP, liposomes. liposome transport study, the TEER values did not obviously reduce $\left(>250 \Omega \mathrm{cm}^{2}\right)$, which indicated that BBB barrier properties were not disrupted during the experiment. As shown in Figure 3, the cumulative cleared volumes of GLU400-LIP, GLU1000-LIP, and GLU2000-LIP were higher than that of GLU200-LIP $(P<0.05)$ and significantly higher than that of the conventional liposome $(P<0.001)$. The cumulative cleared volumes of GLU400-LIP, GLU1000LIP, and GLU2000-LIP were 1.47, 1.39, and 1.45 times higher than that of GLU200-LIP, and 8.10, 7.64, and 7.95 times higher than that of LIP, respectively. That result indicated that glucose-modified liposomes linked by PEGs with longer chain length can promote the drug transport across the BBB barrier. Besides, the transporting process exhibited a time-dependent manner.

\section{Biodistribution in vivo}

\section{In vivo and ex vivo NIR fluorescence imaging}

Recently, NIR fluorescence imaging has been employed widely as a potential tool for qualitative analysis. ${ }^{27,28}$ Light penetrates relatively deeply into the tissue in the NIR wavelength range of 700-900 nm. NIR fluorescence imaging has many advantages such as nonradioactivity and high

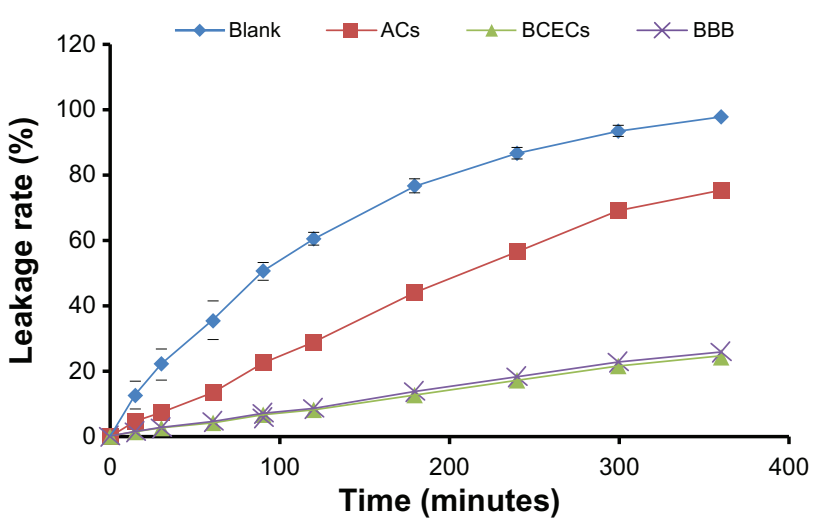

Figure 2 Fluorescein sodium leakage test of the ACs, BCECs, and the BBB model in vitro. The blank inserts were employed as controls $(n=3)$.

Abbreviations: ACs, astrocytes; blood-brain barrier; BCECs, brain capillary endothelial cells. 


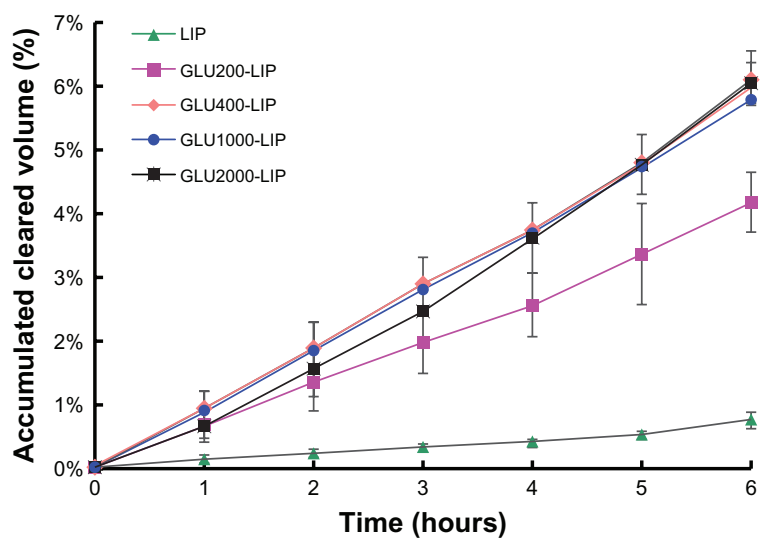

Figure 3 The accumulated cleared volumes of different coumarin 6-loaded liposomes on the blood-brain barrier model in vitro $(n=3)$.

Abbreviations: GLU, glucose; LIP, liposomes.

sensitivity compared with conventional imaging methods. ${ }^{29,30}$ A NIR fluorescence probe DIR was encapsulated in each liposome to trace the liposome behavior in mice. As shown in Figure 4, the signal intensity in the brain of GLU1000-LIP at each time point was all stronger than those of the other groups, which indicated a better brain-targeted property of GLU1000-LIP. As shown in Figure 5, there were NIR fluorescence signals in the excised mouse brains of each group, and the intensity of GLU1000-LIP was the strongest among all the other groups, indicating an increase in the delivery of drug to the brain. Control animals injected with saline solution produced no background signal, which confirms that the observed fluorescence signal was truly from the liposome.

\section{Confocal laser scanning microscopy}

To visualize the ability of the liposomes to transport drug across the BBB barrier into the brain tissues, the frozen sections of mice brains were prepared and imaged accordingly. As shown in Figure 6, GLU1000-LIP revealed the highest accumulation of drug in the brains among all the other groups, validating that GLU1000-LIP had a more significant targeting contribution to the delivery of the drug into the brain.

\section{Quantitative biodistribution study}

To further evaluate the brain-targeted properties of LIP, GLU200-LIP, GLU400-LIP, GLU1000-LIP, and GLU2000LIP, in vivo distribution in mice was studied quantitatively. Coumarin 6 in different tissues could be completely separated and detected using the selected analytical method. For brains, hearts, livers, spleens, lungs, and kidneys, the standard curves were $y=0.006 \times-0.005(0.5-100 \mathrm{ng} / \mathrm{mL})$, $\mathrm{y}=0.011 \times-0.013(0.1-500 \mathrm{ng} / \mathrm{mL}), \mathrm{y}=0.024 \times-2.267$ $(2-15,000 \mathrm{ng} / \mathrm{mL}), \mathrm{y}=0.015 \times+0.017(0.5-1000 \mathrm{ng} / \mathrm{mL})$, $\mathrm{y}=0.009 \times+0.493(1-2000 \mathrm{ng} / \mathrm{mL})$, and $\mathrm{y}=0.007 \times+0.034$ $(5-500 \mathrm{ng} / \mathrm{mL})$, respectively, where y represents the area ratio (coumarin 6/coumarin 7) and $x$ represented the concentration of coumarin $6(\mathrm{ng} / \mathrm{mL})$. $\mathrm{R}^{2}$ remained above 0.997 for all the standard curves, and the recoveries were all between $80 \%-120 \%$. In addition, the within-day precisions and between-day precisions were $4 \%$ and $8 \%$, respectively. As shown in Figure 7, 1 hour after the intravenous injection, the accumulation of GLU1000-LIP in the brains were 3.93, 2.85,

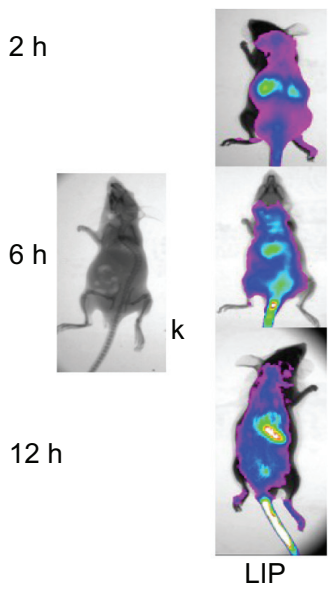

LIP
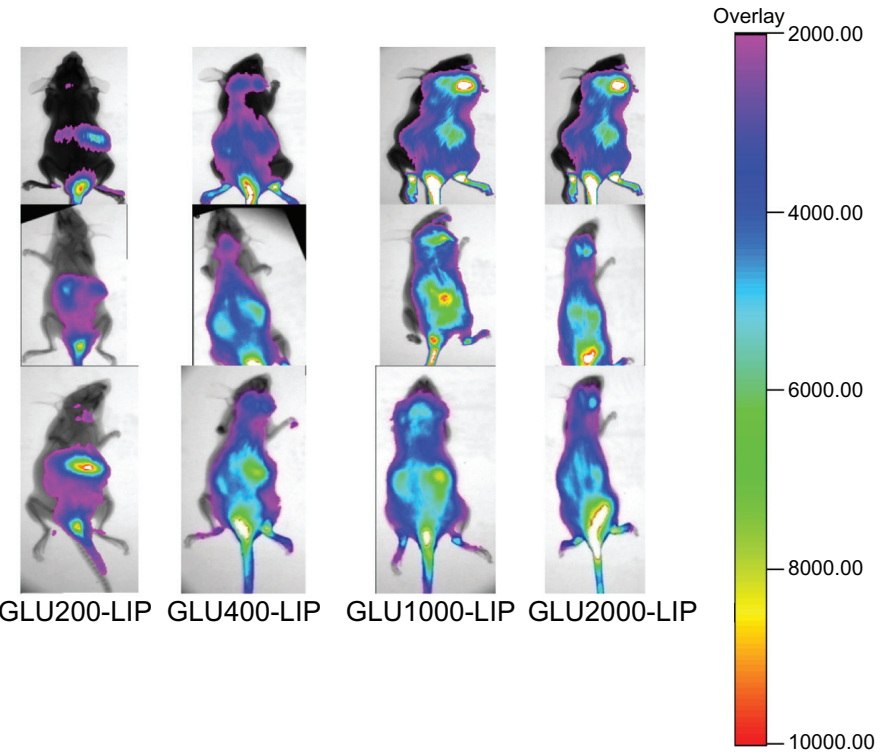

Figure 4 In vivo imaging of the mice that were anesthetized at 2 hours, 6 hours, and 12 hours after intravenous injection of different DIR-loaded liposomes respectively. Notes: Color bar indicates the intensity of the nearinfrared fluorescence signal.

Abbreviations: DIR, I, I'-dioctadecyl-3,3,3',3'-tetramethylindotricarbocyanine; GLU, glucose; LIP, liposomes. 

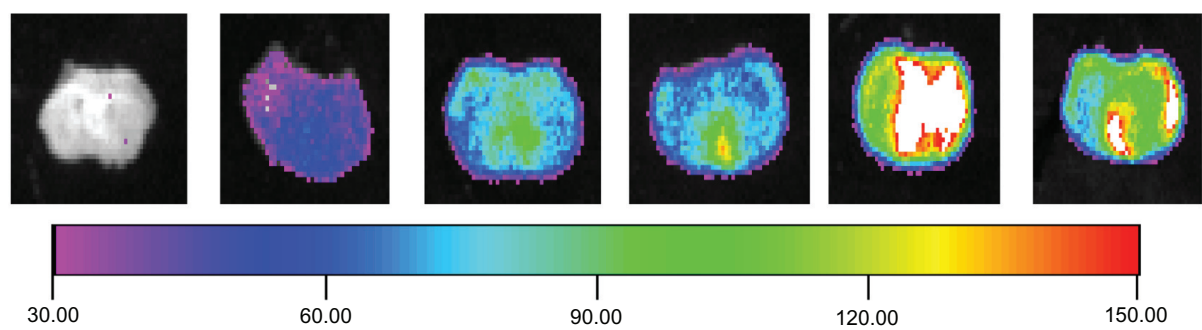

120.00

150.00

Blank

LIP

GLU200-LIP

GLU400-LIP

GLU1000-LIP

GLU2000-LIP

Figure 5 Ex vivo imaging in the mouse brain I hour after the intravenous injection of different DIR-loaded liposomes.

Note: The nearinfrared fluorescence signal intensity is weaker towards the left and stronger towards the right.

Abbreviations: DIR, I, I'-dioctadecyl-3,3,3',3'-tetramethylindotricarbocyanine; GLU, glucose; LIP, liposomes.

2.40, and 1.79 times higher than those of LIP, GLU200-LIP, GLU400-LIP, and GLU2000-LIP, respectively, which indicates a more pronounced targeting efficiency for GLU1000LIP in brain delivery.

\section{Discussion}

Utilization of the carrier systems expressed on the BBB has been an attractive strategy for therapeutic delivery of drugs into the brain, and several carrier-mediated brain-targeting drug-delivery systems have been developed. Particulate drug-delivery systems (such as liposomes and nanoparticles) have been investigated extensively to increase the targeting efficiency of drugs to the brain through the specific endogenous transporters localized on the BBB interacting with the specific ligands modified on the surface of the delivery systems. ${ }^{31,32}$ In this study, we developed a brain-targeted liposomal system by covalently conjugating cholesterol with glucose, as glucose could be transported by GLUTs on the $\mathrm{BBB}$ to reach the action site in the brain.

Liposomes modified by PEGs as the linker between the glucose and the cholesterol obtain long-circulating properties by avoiding the RES effect and decreasing accumulation in the liver and spleen. PEGs also enhanced the chemical stability of the liposomes in serum, and have been applied widely and studied extensively. In bioconjugation modification, PEG has been used repeatedly as a linker by providing functional groups required for conjugation. ${ }^{33} \mathrm{PEG}$ remains as a spacer arm between liposome and conjugated antibody/protein, and the chain length as well as its density could influence the characteristics of the modified vehicles. ${ }^{33}$ End-grafted polymer layers assume uniform chain stretching, where all chain ends are located at the outer brush edge $\mathrm{e}^{34}$ and could be conjugated with ligand for targeting purposes; in our case it was glucose. Therefore, a PEG spacer long enough to ensure the sufficient exposure of the ligands on the liposome is of great importance. On the other hand, PEGs with a chain length that is too long are more flexible and tend to fold together, which could also reduce the exposure of the ligands. Also, the steric hindrance could take effect and block the interactions between liposomes and targeted sites. To maintain this subtle balance and obtain the optimum formulation, modifications of PEGs with different chain lengths were designed and investigated.

PEGylation and ligand-modification were often performed on phospholipids which served as one of the components in the liposomes. Another important component is cholesterol. However, it has always being neglected as an alternative anchor. Cholesterol as an anchor may own some merits compared with 1,2-dioctadecanoyl-sn-glycero-3-phosphoethanolamine (DSPE). Firstly, DSPE covalently conjugated to certain ligands would introduce a negative charge to the liposome surface, thus inducing additional plasma protein-binding.

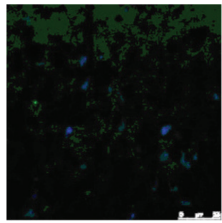

LIP

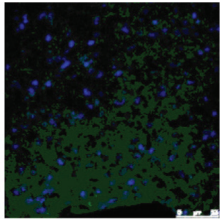

GLU200-LIP

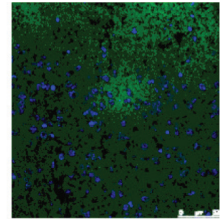

GLU400-LIP

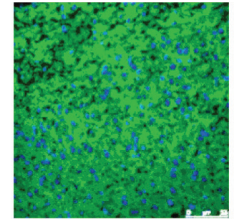

GLU1000-LIP

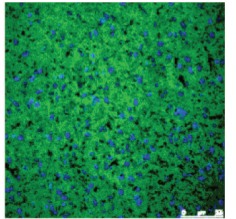

GLU2000-LIP

Figure 6 Confocal laser scanning microscopy images of the mouse brain frozen sections I hour after the intravenous injection of different coumarin 6-loaded liposomes. Note: The scale bar is $75 \mu \mathrm{m}$ on the lower right corner in each image.

Abbreviations: GLU, glucose; LIP, liposomes. 
In contrast, cholesterol, as an alternative anchor, is electrically neutral. Secondly, the DSPE molecule is not as chemically stable as cholesterol and has the tendency to degrade during storage and application. Thirdly, the cost of DSPE is more than 100 times higher than that of cholesterol, which is less desirable for product development. The replacement of DSPE with cholesterol is potentially more practical. ${ }^{35}$

Particle size plays a critical role in their clearance by the sinusoidal spleens of human and rats. Particles must be small enough to avoid the splenic filtration process at the interendothelial cell slits (IES) in the walls of venous sinuses. ${ }^{36}$
Similarly, particle size is an important factor that affects the liposome endocytosis by the brain capillary cells on the $\mathrm{BBB}$, and the size distribution is generally limited within $200 \mathrm{~nm}$ in diameter for brain-targeted liposomes. ${ }^{37}$ In our study, the sizes of the prepared liposomes were all below $170 \mathrm{~nm}$, which provided a favorable size condition for brain transport. The particle sizes decreased due to the stabilizing effect of PEGs which hindered the liposome interactions. The PDIs increased with the introduction of PEGs, which can be explained by the greater flexibility and folding of longer chains. ${ }^{38}$ In our study, PDIs less than 0.300 and
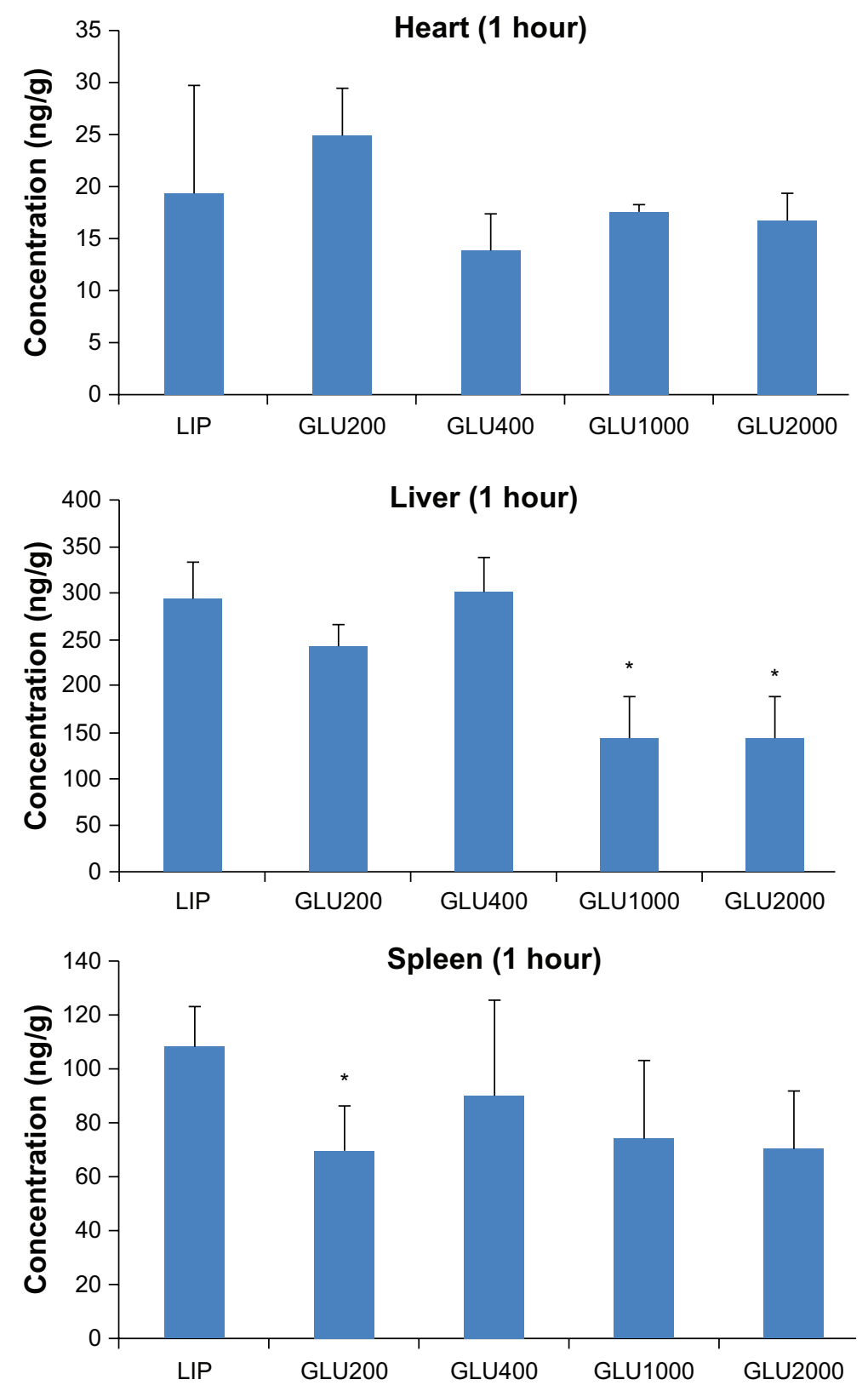

Figure 7 (Continued) 

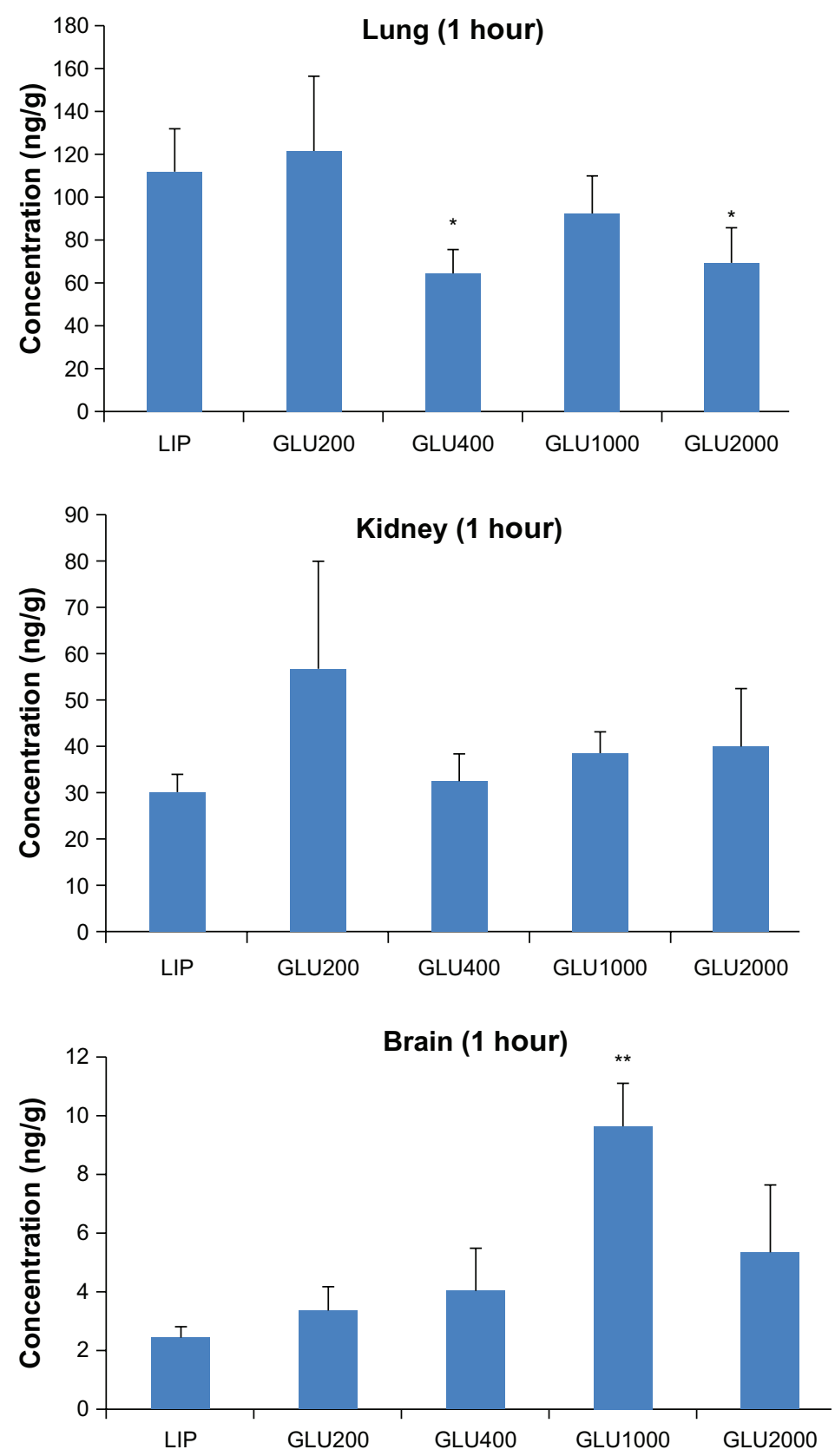

Figure 7 The distributions in different organs I hour after the intravenous injection of different coumarin 6-loaded liposomes $(\mathrm{n}=3$ ). Notes: $* P<0.05$ compared with LIP; **P $<0.0$ I compared with LIP; ***P $<0.00$ I. compared with LIP.

Abbreviations: GLU, glucose; LIP, liposomes.

particle diameters within $200 \mathrm{~nm}$ were considered adequate for further in vitro and in vivo studies. ${ }^{39,40,42}$

After intravenous injection, the formulation could adsorb onto the plasma protein to form large particles. The large particles can be taken up by the RES readily, impeding the sufficient delivery of the formulation to the action site. The stabilities of LIP, GLU200-LIP, GLU400-LIP, GLU1000LIP, and GLU2000-LIP were investigated to evaluate the possibility of the formulations reaching the targeted site.
The transmittancy of each sample incubated with $10 \%$ FBS was detected at different time points during a total of 24 hours. The variations in the transmittances of the four PEG-modified glucose-linked liposomes (GLU200-LIP, GLU400-LIP, GLU1000-LIP, and GLU2000-LIP) were not as significant as the conventional group (LIP). No drastic increase in turbidity suggested that these formulations might not generate a large aggregate in bloodstream and could maintain their stabilities in vivo. ${ }^{41}$ On the contrary, the 
absence of PEGs on the surface of LIP led to the formation of conglomeration and an reduction of the transmittance. Also, the absolute value of the zeta-potential of LIP increased without the sheltering of PEG chain coatings, contributing to its decreased stability.

Tight junctions between the BCECs play an important role in maintaining the barrier properties of the BBB in vitro. The results of the fluorescein sodium leakage test certified the reliability and integrity of the BBB model in vitro. Furthermore, the TEER value of the coculture remained above $250 \Omega \mathrm{cm}^{2}$ from the beginning to the end during the experiment, which indicated that the liposome transport did not violate the integrity of the BBB barrier. ${ }^{43}$ As illustrated in Figure 3, the results showed that the uptake of GLU400-LIP, GLU1000-LIP, and GLU2000LIP was more efficient than that of GLU200-LIP by the BBB in vitro, and the transendothelial abilities of the four types of glucose-modified liposomes were all much stronger than that of LIP, which is evidence that glucose-modification and longer PEGs could promote the liposome to transport across the $\mathrm{BBB}$. The results also confirmed that PEGs with shorter chain length (PEG 200) may obstruct the exposure of the glucose. Liposomes modified with longer PEGs (PEG400, PEG1000, and PEG2000) displayed similar profiles on the in vitro BBB models. This might be because the in vitro BBB model cannot entirely imitate in vivo conditions, so the long-circulating effects of PEG-modified liposomes did not differ from each other. Due to the sophistication of in vivo environments and to further confirm the behaviors of different formulations upon entering the circulation, in vivo experiments were conducted to determine the influences of PEGs with different chain lengths on the delivery properties of the liposomes.

The ex vivo and in vivo NIR fluorescent imaging and quantitative biodistribution study could reveal the braintargeted properties of each formulation in vivo more scientifically and directly. Compared with other organs, especially livers and spleens, the amount of the liposomes delivered to the brains is not that much higher. BBB as a natural barrier poses a formidable hazard for the fabrication of brain-targeted drug-delivery systems. However, the developed formulation achieved a greatly improved drug accumulation in the brains compared with conventional formulation. As shown in Figure 7, the accumulation in the livers was obviously reduced in GLU1000-LIP and GLU2000-LIP, which demonstrates an evident long-circulating effect endowed by the liposomes with longer PEGs as the linker. Also, it was obvious that GLU1000-LIP accumulated the most in the brains 1 hour after injection, which displays a stronger brain-targeted capacity compared with the other groups. Harashima and colleagues ${ }^{44}$ argued that shortening the PEG chain to a proper length would result in a facilitated membrane fusion between the lipid envelope and the endosomal membrane, and they found PEG900 as the linker produced higher transfection efficiency than PEG2000. This conclusion was further supported by our study that PEG1000 (the chain length of which was close to PEG900) could realize the best targeted delivery among all the different PEG modifications including PEG2000 and others with shorter chain length.

\section{Conclusion}

The experimental data in vitro and in vivo indicated that the glucose-modified liposomes using PEGs with longer chain length as the linker was a promising brain-targeted drug-delivery system due to its high delivery efficiency across the BBB. Among all the experimental groups, GLU1000-LIP exhibited the strongest brain delivery efficiency, proving itself to be a potential brain-targeted drug-delivery system.

\section{Acknowledgments}

We are thankful for the financial support of the State Key Program of National Natural Science of China (81130060), and the National Basic Research Program (2009CB903300). We acknowledge Chunmeng Shi and Yongping Su from the Institute of Combined Injury in the Third Military Medical University for providing equipment for the optical imaging.

\section{Disclosure}

The authors declare they have no competing interests in this work.

\section{References}

1. Pardridge WM. Blood-brain barrier biology and methodology. J Neuroviro. 1999;5(6):556-569.

2. Pardridge WM. Drug and gene targeting to the brain via blood-brain barrier receptor-mediated transport systems. Int Congr. 2005;1277:49-62.

3. Rubin LL, Staddon JM. The cell biology of the blood-brain barrier. Annu Rev Neurosci. 1999;22:11-28.

4. Cardoso FL, Brites D, Brito MA. Looking at the blood-brain barrier: Molecular anatomy and possible investigation approaches. Brain Res Rev. 2010;64(2):328-363.

5. Begley DJ, Brightman MW. Structural and functional aspects of the blood-brain barrier. Prog Drug Res. 2003;61:41-78.

6. Polt R, Porreca F, Szabo LZ, et al. Glycopeptide enkephalin analogues produce analgesia in mice: evidence for penetration of the blood-brain barrier. Proc Natl Acad Sci U S A. 1994;91(15):7114-7118.

7. Halmos T, Santarromana M, Antonakis K, Scherman D. Synthesis of O-methylsulfonyl derivatives of D-glucose as potential alkylating agents for targeted drug delivery to the brain. Evaluation of their interaction with the human erythrocyte GLUT1 hexose transporter. Carbohydr Res. 1997;299(1-2):15-21.

8. Zhang ZR, Luo WZ, Nagai T. Studies on hydrolysis kinetics and photolysis kinetics of N1-retinoyl-5-fluorouracil. STP Pharma Sci. 2001;11(3): 243-246. 
9. Bonina FP, Arenare L, Ippolito R, et al. Synthesis, pharmacokinetics and anticonvulsant activity of 7-chlorokynurenic acid prodrugs. Int $J$ Pharm. 2000;202(1-2):79-88.

10. Huang DW, Shen SC, Wu JS. Effects of caffeic acid and cinnamic acid on glucose uptake in insulin-resistant mouse hepatocytes. J Agrc Food Chem. 2009;57(17):7687-7692.

11. Gould GW, Holman GD. The glucose transporter family: structure, function and tissue-specific expression. Biochem J. 1993;295(2):329-341.

12. Schubert D. Glucose metabolism and Alzheimer's disease. Ageing Res Rev. 2005;4(2):240-257.

13. Qutub AA, Hunt CA. Glucose transport to the brain: a systems model. Brain Res Rev. 2005;49(3):595-617.

14. Dwyer DS, Vannucci SJ, Simpson IA. Expression, regulation, and functional role of glucose transporters (GLUTs) in brain. Int Rev Neurobiol. 2002;51:159-188.

15. Regina A, Roux F, Revest PA. Glucose transport in immortalized rat brain capillary endothelial cells in vitro: transport activity and GLUT1 expression. Biochim Biophys Acta. 1997;1335(1-2):135-143.

16. McAllister MS, Krizanac-Bengez L, Macchia F, et al. Mechanisms of glucose transport at the blood-brain barrier: an in vitro study. Brain Res. 2001;904(1):20-30.

17. Vakil R, Kwon GS. PEG-phospholipid micelles for the delivery of amphotericin B. J Control Release. 2005;101(1-3):386-389.

18. Torchilin VP, Trubetskoy VS, Whiteman KR, et al. New synthetic amphiphilic polymers for steric protection of liposomes in vivo. J Pharm Sci. 1995;84(9):1049-1053.

19. Yamamoto Y, Nagasaki Y, Kato Y, et al. Long-circulating poly (ethylene glycol)-poly (D,L-lactide) block copolymer micelles with modulated surface charge. J Control Release. 2001;77(1-2):27-38.

20. Qin Y, Fan W, Chen H, et al. In vitro and in vivo investigation of Glucose-mediated brain-targeting liposomes. J Drug Target. 2010;18(7):536-549

21. Lei F, Fan W, Li XK, et al. Design, synthesis and preliminary bioevaluation of glucose-cholesterol derivatives as ligands for brain targeting liposomes. Chinese Chem Lett. 2011;22(7):831-834.

22. Zhao XB, Muthusamy N, Byrd JC, et al. Erratum: Cholesterol as a bilayer anchor for PEGylation and targeting ligand in folate-receptortargeted liposomes. J Pharm Sci. 2007;96(9):2424-2439.

23. Lu W, Tan YZ, Hu KL, et al. Cationic albumin conjugated pegylated nanoparticle with its transcytosis ability and little toxicity against bloodbrain barrier. Int J Pharm. 2005;295(1-2):247-260.

24. Lu W, Zhang Y, Tan YZ, et al. Cationic albumin-conjugated pegylated nanoparticles as novel drug carrier for brain delivery. J Control Release. 2005;107(3):428-448.

25. Demeuse P, Kerkhofs A, Struys-Ponsar C, et al. Compartmentalized coculture of rat brain endothelial cells and astrocytes: a syngenic model to study the blood-brain barrier. J Neurosci Methods. 2002; 121(1):21-31

26. Hayashi Y, Nomura M, Yamagishi S, et al. Induction of various bloodbrain barrier properties in non-neural endothelial cells by close apposition to co-cultured astrocytes. Glia. 1997;19(1):13-26.

27. Zhang Y, Schlachetzki F, Zhang YF, et al. Normalization of striatal tyrosine hydroxylase and reversal of motor impairment in experimental parkinsonism with intravenous nonviral gene therapy and a brainspecific promoter. Hum Gene Ther. 2004;15(4):339-350.
28. Ripolles T, Agramunt M, Martinez MJ, et al. The role of ultrasound in the diagnosis, management and evolutive prognosis of acute left-sided colonic diverticulitis: a review of 208 patients. Eur Radiol. 2003;13(12):2587-2595.

29. Frangioni JV. In vivo near-infrared fluorescence imaging. Curr Opin Chem Biol. 2003;7(5):626-634.

30. Xu RX, Young DC, Mao JJ, Povoski SP. A prospective pilot clinical trial evaluating the utility of a dynamic near-infrared imaging device for characterizing suspicious breast lesions. Breast Cancer Res. 2007;9(6):R88.

31. Tromberg BJ, Pogue BW, Paulsen KD, et al. Assessing the future of diffuse optical imaging technologies for breast cancer management. Med Phys. 2008;35(6):2443-2451.

32. Pardridge WM. Blood-brain barrier drug targeting: the future of brain drug development. Mol Interv. 2003;3(2):90-105.

33. Cruz LJ, Tacken PJ, Fokkink R, Figdor CG. The influence of PEG chain length and targeting moiety on antibody-mediated delivery of nanoparticle vaccines to human dendritic cells. Biomaterials 2011;32(28):6791-6803.

34. Manjappa AS, Chaudhari KR, Venkataraju MP, et al. Antibody derivatization and conjugation strategies: application in preparation of stealth immunoliposome to target chemotherapeutics to tumor. $J$ Control Release. 2011;150(1):2-22.

35. Lasic DD. Doxorubicin in sterically stabilized liposomes. Nature. 1996;380(6574):561-562.

36. Han HD, Lee A, Song CK, et al. In vivo distribution and antitumor activity of heparin-stabilized doxorubicin-loaded liposomes. Int $J$ Pharm. 2006;313(1-2):181-188.

37. Kaur IP, Bhandari R, Bhandari S, Kakkar V. Potential of solid lipid nanoparticles in brain targeting. J Control Release. 2008;127(2):97-109.

38. Du J, Lu WL, Ying X, et al. Dual-targeting topotecan liposomes modified with tamoxifen and wheat germ agglutinin significantly improve drug transport across the blood-brain barrier and survival of brain tumor-bearing animals. Mol Pharm. 2009;6(3):905-917.

39. Shimada K, Kamps JA, Regts J, et al. Biodistribution of liposomes containing synthetic galactose-terminated diacylglyceryl-poly(ethyleneglycol)s. Biochim Biophys Acta. 1997;1326(2):329-341.

40. Costanzo PJ, Dan N, Lancaster KS, et al. Effect of changing polymer chain length on the target-mediated agglutination of polymer-grafted nanoparticles. Macromolecules. 2008;41(4):1570-1576.

41. Maeda N, Takeuchi Y, Takada M, et al. Anti-neovascular therapy by use of tumor neovasculature-targeted long-circulating liposome. J Control Release. 2004;100(1):41-52.

42. Pietkiewicz J, Zielinska K, Saczko J, et al. New approach to hydrophobic cyanine-type photosensitizer delivery using polymeric oil-cored nanocarriers: Hemolytic activity, in vitro cytotoxicity and localization in cancer cells. Eur J Pharm Sci. 2010;39(5):322-325.

43. Ying X, Wen H, Lu WL, et al. Dual-targeting daunorubicin liposomes improve the therapeutic efficacy of brain glioma in animals. J Control Release. 2010;141(2):183-192.

44. Ishitsuka T, Akita H, Harashima H. Functional improvement of an IRQ-PEG-MEND for delivering genes to the lung. J Control Release. 2011;154(1):77-83.

International Journal of Nanomedicine

\section{Publish your work in this journal}

The International Journal of Nanomedicine is an international, peerreviewed journal focusing on the application of nanotechnology in diagnostics, therapeutics, and drug delivery systems throughou the biomedical field. This journal is indexed on PubMed Central,

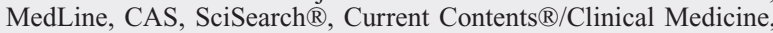

Journal Citation Reports/Science Edition, EMBase, Scopus and the Elsevier Bibliographic databases. The manuscript management system is completely online and includes a very quick and fair peer-review system, which is all easy to use. Visit http://www.dovepress.com/ testimonials.php to read real quotes from published authors. 